\title{
Manobras de reexpansão pulmonar no pós-operatório de cirurgia cardíaca: revisão bibliográfica
}

\author{
Pulmonary reexpansion maneuvers in the postoperative period of cardiac surgery: literature \\ review
}

Maniobras de reexpansión pulmonar em el postoperatorio de cirugía cardíaxa: revisión de literatura

\author{
Willsyany Monteiro Assunção Dias \\ ORCID: https://orcid.org/0000-0002-3414-071X \\ Instituto Educacional Santa Catarina-Faculdade Guaraí, Brasil \\ E-mail: willsyany.monteiro@gmail.com \\ Rafaela Ferreira Da Silva \\ ORCID: https://orcid.org/0000-0002-2022-8366 \\ Instituto Educacional Santa Catarina-Faculdade Guaraí, Brasil \\ E-mail: rafaelaferreira241199@gmail.com \\ Lécia Kristine Lourenço \\ ORCID: https://orcid.org/0000-0002-0531-7221 \\ Instituto Educacional Santa Catarina-Faculdade Guaraí, Brasil \\ E-mail: leciakristine@yahoo.com.br
}

\begin{abstract}
Resumo
A cirurgia cardíaca é um processo de restauração desde que a capacidade vital seja compatível com a capacidade funcional do sistema cardíaco respiratório, devem ser realizadas quando não é alcançado um resultado positivo no tratamento conservador aos pacientes com disfunções cardíacas, não melhorando sua função e nem permitindo o seu retorno as condições físicas e sociais, A fisioterapia respiratória atua na aplicação de inúmeras técnicas que previnem complicações pulmonares como: retenção de líquidos e secreções, atelectasias e pneumonia. Objetivo: O objetivo geral deste estudo é verificar as principais complicações que estão relacionadas a função pulmonar evidenciando as condutas de expansão pulmonar dentre as terapias utilizadas pelo fisioterapeuta. Metodologia: Para realização deste trabalho, foi executado um levantamento bibliográfico, acerca do assunto referente as manobras expansivas em pacientes submetidos a cirurgia torácica e abdominal na base de dados: LILACS (Literatura Latino-americana em Ciências da saúde), SCIELO (Scientific Eletronic Library Online), google acadêmico e livros, utilizando as palavras chaves: fisioterapia respiratória, reexpansão pulmonar, cirurgia cardíaca. Conclusão: O presente estudo demonstra que a cirurgia cardíaca provoca várias alterações pulmonares, renais, neurológicas, sendo comum ocorrer cerca de $70 \%$ dos casos. De acordo com os estudos analisados, a aplicação das técnicas de reexpansão pulmonar no pós-operatório de cirurgia cardíaca se mostrou relevante na melhora do quadro funcional desses pacientes.
\end{abstract}

Palavras-chave: Cirurgia cardíaca; Complicações pós-cirúrgicas; Fisioterapia respiratória; Reexpansão pulmonar.

\begin{abstract}
Cardiac surgery is a restoration process as long as the vital capacity is compatible with the functional capacity of the respiratory cardiac system, it must be performed when a positive result is not achieved in the conservative treatment of patients with cardiac dysfunction, not improving its function and not even allowing its return to physical and social conditions. Respiratory physiotherapy pulmo with the application of numerous techniques that prevent pulmonar complications such as: retention of fluids and secretions, atelectasis and pneumonia. Objective: The general objective of this study is to verify the main complications that are related to lung function, evidencing lung expansion approaches among the therapies used by physical therapists. Methodology: To carry out this work, a bibliographic was carried out on the subject of expansive maneuvers in patients undergoing thoracic and abdominal surgery in the database: LILACS (Latin American Literature in Health Sciences), SCIELO (Scientific Electronic Library Online), academic google and books, using the pulmonar: respiratory physiotherapy, lung reexpansion, cardiac surgery. Conclusion: The pulmonar study demonstrates that cardiac surgery causes several pulmonar, renal, neurological changes, being common to occur in about $70 \%$ of cases. According to the studies analyzed, the application of pulmonar re-expansion techniques in the postoperative period of cardiac surgery proved to be relevant in improving the functional status of these patients.
\end{abstract}

Keywords: Cardiac surgery; Post-surgical complications; Respiratory physiotherapy; Lung reexpansion. 


\begin{abstract}
Resumen
La cirugía cardíaca es un proceso reparador, siempre que la capacidad vital sea compatible con la capacidad funcional del sistema respiratorio cardíaco, se debe realizar cuando no exista resultado positivo en el tratamiento conservador de pacientes con disfunción cardíaca, no mejorando su La fisioterapia respiratoria trabaja con la aplicación de numerosas técnicas que previenen las complicaciones pulmonares como: retención de líquidos y secreciones, atelectasias y neumonías. Objetivo: el objetivo general de este estudio es verificar las principales complicaciones relacionadas con la función pulmonar, mostrando comportamientos de expansión pulmonar entre las terapias utilizadas por los fisioterapeutas. Metodología: Para la realización de este trabajo se realizó un relevamiento bibliográfico sobre el tema de maniobras expansivas en pacientes sometidos a cirugía torácica y abdominal en la base de datos: LILACS (Literatura Latinoamericana en Ciencias de la Salud), SCIELO (Electronic Scientific Library Online), google académico y libros, utilizando las palabras clave: fisioterapia respiratoria, reexpansión pulmonar, cirugía cardíaca. Conclusión: El presente estudio muestra que la cirugía cardíaca ocasiona varios cambios pulmonares, renales y neurológicos, siendo común que ocurra en alrededor del 70\% de los casos. Según los estudios analizados, la aplicación de técnicas de reexpansión pulmonar en el postoperatorio de cirugía cardíaca resultó ser relevante para mejorar el estado funcional de estos pacientes.
\end{abstract}

Palabras clave: Cirugía cardíaca; Complicaciones posquirúrgicas; Fisioterapia respiratoria; Reexpansión pulmonar.

\title{
1. Introdução
}

As doenças cardiovasculares estão entre as relevantes causas de morte no mundo desenvolvido e sua ocorrência tem crescido de forma preocupante, no contexto brasileiro observa-se 32,6\% de mortalidade por causas especificas, liderando a ocupação dos leitos hospitalares (Renault, et.al. 2008).

Para Morais (2010), as cirurgias cardíacas é um processo de restauração desde que a capacidade vital seja compatível com a capacidade funcional do sistema cardíaco respiratório, devem ser realizadas quando não é alcançado um resultado positivo no tratamento conservador aos pacientes com disfunções cardíacas, não melhorando sua função e nem permitindo o seu retorno as condições físicas e sociais.

As cirurgias cardíacas tem efeito evidente no mecanismo respiratório, que colabora iminentemente para o desenvolvimento de complicações pulmonares, como hipoxemia e atelectasia, além de outros fatores como a dor, reduz a efetividade da tosse e inibi a capacidade do paciente realizar uma inspiração profunda, favorecendo o acumulo de secreções, como também o local da incisão, os drenos colocados e a anestesia são fatores que influenciam diretamente na função pulmonar, dificultando a expansibilidade. Além disso o tempo de internação hospitalar frequentemente causa estresse, incomodidade, exacerbação da ansiedade e consequentemente da dor, afetando o transporte de $\mathrm{O} 2$ contribuindo para o desenvolvimento de complicações no pós-operatório (Azeredo, et al., 2002).

A fisioterapia respiratória atua na aplicação de inúmeras técnicas que previnem complicações pulmonares como: retenção de líquidos e secreções, atelectasias e pneumonia. O período e frequência da fisioterapia respiratória para pacientes cirúrgicos são variadas, dependendo das necessidades individuais, preferência terapêutica e prática institucional. Apesar da falta de evidencias de que os exercícios respiratórios diminuem as complicações pulmonares, as manobras de reexpansão pulmonares são amplamente aplicadas nas fases de pré e pós-operatório de cirurgias abdominais e torácicas. As manobras de reexpansão pulmonar correspondem ao tratamento que estimula o paciente a efetuar inspirações mais profundas (Cavenaghi, et al., 2011). São associados os movimentos de membros superiores e mudanças de decúbito associadas ao ciclo respiratório, pode-se utilizar dispositivos que auxiliam no aumento do volume inspirado e na manutenção de uma pressão positiva no final da expiração, dificultando o colapso alveolar. Durante a reexpansão pulmonar acontecem mudanças nos volumes pulmonares consequentemente causados por mudanças nas pressões do sistema respiratório, particularmente nas pressões pleural e alveolar (Cavenaghi, et al., 2011).

Para a execução das técnicas de reexpansão pulmonar, deve ser levado em consideração a semiologia do tórax, ausculta pulmonar, radiografia de tórax, gasometria arterial e eletrocardiograma, também deve ser definido o posicionamento apropriado 
para o paciente, além de ter conhecimento fisiológico, para a realização do posicionamento que traga mais benefícios durante a realização das técnicas, uma vez que pode induzir no resultado. Os métodos de reexpansão pulmonar são mais indicadas quando ocorre redução dos volumes pulmonares e/ou quando há risco de colapso alveolar. Sendo assim deve ser considerado realizar reexpansão pulmonar quando há condições restritivas como: atelectasias, fibrose pulmonar, derrames pleurais, deformidades torácicas e obesidade (Lima, et al.,2011).

É importante destacar que cooperação do paciente, assim como a orientação antes do procedimento é de suma importância para a eficácia do exercício. O ajustamento da duração do tempo inspiratório de expiratório, a conscientização da utilização da musculatura respiratória e um bom comando verbal é necessário para a efetividade das técnicas, sendo assim o objetivo deste estudo é verificar as principais complicações relacionadas a função pulmonar no pós-operatório cirurgia cardíaca evidenciando as condutas de expansão pulmonar dentre as terapias utilizadas pelo fisioterapeuta (Renault, et.al. 2008).

\section{Metodologia}

A presente pesquisa bibliográfica tem como finalidade descrever e compartilhar conhecimentos sobre as principais técnicas de reexpansão pulmonar e a atuação do profissional fisioterapeuta na aplicação dessas manobras.

A pesquisa bibliográfica é o levantamento ou revisão de obras publicadas sobre a teoria que irá direcionar o trabalho científico o que necessita uma dedicação, estudo e análise pelo pesquisador que irá executar o trabalho científico e tem como objetivo reunir e analisar textos publicados, para apoiar o trabalho científico. Para Gil (2002), a pesquisa bibliográfica é desenvolvida com base em material já elaborado, constituído principalmente de livros e artigos científicos.

A pesquisa é fundamentada no referencial bibliográfico, a literatura cientifica utilizada tem origem no idioma língua portuguesa, inglesa e espanhola, sendo considerada nesse estudo, livros, artigos e trabalhos publicados.

Para realização deste trabalho, foi executado um levantamento bibliográfico, acerca do assunto referente as manobras expansivas em pacientes submetidos a cirurgia torácica e abdominal na base de dados: LILACS (Literatura Latino-americana em Ciências da saúde), SCIELO (Scientific Eletronic Library Online), google acadêmico e livros, utilizando as palavras chaves: fisioterapia respiratória, reexpansão pulmonar, cirurgia cardíaca.

\section{Resultados e Discussão}

\subsection{Atuação do Fisioterapeuta nas manobras de Reexpansão Pulmonar}

A intervenção cirúrgica é bastante complexa e tem importantes impactos na homeostase corporal, alterando o funcionamento fisiológico do paciente, podendo ocasionar na necessidade de cuidados intensivos com intuito de estabelecer uma boa recuperação do paciente (Dias, et al., 2011). A falta de cuidados durante o período pós-operatório pode acarretar afecções, surgindo sequelas graves ou até óbito do paciente. A cirurgia cardíaca tem por característica a abertura do tórax e abdômen, esta abertura modifica todas as pressões internas existentes na região (Pryor, et al.,2002). Por isso, os exercícios respiratórios são de suma importância, pois previnem e amenizam as alterações pulmonares, melhora na capacidade física global, evitando pneumonias, atelectasias e outros problemas. Ao surgirem essas complicações durante esse período de internação deve-se ser identificada e corrigida mais rapidamente, a fim de estabelecer condutas para reverter o quadro desse paciente, desta forma, a fisioterapia no pós-operatório tem como principais objetivos a reexpansão pulmonar, a desobstrução das vias aéreas e orientar os responsáveis pela prevenção de complicações.

“Pacientes que não são estimulados a modificar o volume corrente pulmonar podem reter e acumular secreção. Sem o estímulo da tosse - que muitas vezes os pacientes evitam por medo - a secreção se acumula e pode provocar atelectasia, que é o fechamento de áreas pulmonares.” - Fisioterapeuta Voldiana L. Pozzebon Schneider (CREFITO 80155/F). 
A fisioterapia vem sendo uma recomendação importante no pós-operatório de cirurgia cardíaca, e os hospitais vêm cada vez mais adotando em suas práticas. Em muitos casos, os pacientes submetidos a cirurgia cardíacas, tem capacidade para respirar normalmente, mas, por receio, acabam realizando uma respiração muito superficial. Ter um fisioterapeuta na equipe multidisciplinar, auxiliando na respiração após a cirurgia cardíaca, proporciona ao paciente mais segurança no retorno volta das atividades de vida normais, além de diminuir a permanência desse paciente no leito hospitalar (Tarantino, et al., 2002). O modo, a frequência e a duração dos atendimentos, devem ser avaliados em cada caso, de acordo com a necessidade individual de cada paciente. Em alguns pacientes, as intervenções serão feitas de forma ativa, já em outros de forma passiva. Más em todos os pósoperatórios de cirurgia cardíaca são recomendados que os atendimentos sejam iniciados em menos de 12 horas após o procedimento (Peixoto, et al., 2009). Exercícios e manobras de reexpansão pulmonar, durante a hospitalização, trazem melhoras na oxigenação e ajudam no mecanismo de tosse, além de melhorar a troca gasosa, aumentando complacência pulmonar e fornecendo uma ventilação adequada para o funcionamento do corpo além de garantir a higiene dos brônquios e atuando de forma a prevenir as complicações (Corrêa, et al., 2009). Dentre as diversas técnicas e condutas a ser realizadas, trouxemos nesse trabalho as principais manobras de reexpanção pulmonar dentre as quais mais se destacaram e trouxeram benefícios em pacientes submetidos a cirurgia cardíaca.

\subsection{Reexpansão pulmonar}

As indicações para a aplicação das manobras de reexpansão pulmonar são quaisquer condições que diminuem volumétricamente a expansão pulmonar, incapacitando o paciente de realizar uma respiração profunda. Todos os tipos de terapia de reexpansão visam aumentar o volume pulmonar por meio do aumento do gradiente de pressão transpulmonar (PT) é a diferença entre as duas pressões acima, ou seja, é a diferença de pressão entre o interior dos alvéolos e a superfície do pulmão. Quanto maior o gradiente de pressão transpulmonar, maior a expansão alveolar. O foco da execução das manobras fisioterapêuticas é selecionado de acordo com a avaliação do paciente sendo assim a escolha das técnicas deve ser baseada de acordo com o diagnostico funcional, tendo maior eficácia em pacientes colaborativos e capazes de gerar grandes volumes pulmonares respeitando o nível de consciência e colaboração do paciente.

\subsection{Exercícios respiratórios diafragmáticos}

A ação do diafragma é de grande importância na ventilação pulmonar. O diafragma tem $70 \%$ na participação do volume corrente, sendo assim o principal musculo responsável pela inspiração. Deve-se orientar ao paciente a ficar com o tórax em posição vertical (sentado), certificando aumento de ventilação nas zonas basais pulmonares (Azeredo et, al.,2002). O paciente deve ser orientado a executar uma inspiração nasal profunda juntamente com uma expiração usando técnica de freno labial. Durante a fase de inspiração ocorre a excursão diafragmática, obtida por uma contração voluntaria. Esse exercício é indicado para pacientes com complacência pulmonar reduzida, permitindo maior expansão pulmonar por aumento da ventilação nas bases. Os exercícios respiratórios diafragmáticos diminuem consideravelmente a atelectasia e melhoram os valores espirometricos no pós-operatório de miocárdio (Vera lucia, et al., 2006).

\subsection{Exercícios respiratórios com inspiração máxima sustentada}

É a realização de uma inspiração nasal lenta, profunda e uniforme seguida de apneia pós-inspiratórios (que deve ser sustentada por um intervalo de tempo mensurado em segundos ao paciente). Em Seguida solicitar ao paciente que realiza uma expiração lente e sem esforço até o volume de reserva expiratório. O objetivo deste exercício é que a apneia pós-inspiratória 
sustentada melhore a distribuição do volume em unidades com baixa complacência e alta resistência, permitindo assim uma ventilação mais uniforme (Vera lucia, et al., 2006).

\subsection{Exercícios de inspiração fracionada}

A inspiração fracionada ou em tempos, é efetiva na melhora da complacência taracopulmonar, tendo contraindicações quando há aumento da resistência das vias aéreas, pois pode haver a hiperinflação pulmonar e o aumento do trabalho respiratório. A inspiração fracionada consiste na realização de inspirações nasais, curtas, suaves e programadas de dois a seis tempos, por períodos de apneia pós inspiratórios seguida de uma expiração oral até o volume expiratório de repouso, as inspirações devem ser realizadas dentro do mesmo ciclo respiratório (Guizilini, et al., 2006).

\subsection{Exercícios de respiração abreviada}

Exercício que utiliza inspirações fracionadas, intercaladas por breves expirações até que atinja a capacidade pulmonar total (CPT), essa técnica resume-se em realizar ciclos intermitentes de inspirações profundas alternadas com expirações curtas, iniciando com uma inspiração nasal lenta e profunda até a CPT, logo após realiza-se novamente uma inspiração até a CPT, seguida de outra expiração curta. Essa técnica deve ser realizada de três a quatro vezes, em seguida realizar uma respiração completa. Esse exercício é contraindicado em situações de aumento da resistência nas vias aéreas por favorecer a hiperinsuflação pulmonar. A execução desta técnica se mostrou efetiva em aumentar a ventilação das zonas pulmonares dependentes (Vera Lucia. et al., 2006).

\subsection{Respirações com pressão positiva intermitente (RPPI)}

A RPPI é baseada na utilização na pressão positiva inspiratória na respiração espontânea, é conduta fisioterapêutica a curto ou em longo prazo, ou seja, é definida como forma de exercício respiratório aplicando pressão positiva para aumento dos volumes pulmonares e a capacidade vital do paciente. Tem como objetivo aplicar pressão positiva na fase inspiratória levando o aumento da pressão alveolar aumentando a capacidade inspiratória. A técnica é realizada através dos ajustes via máscara facial ou bucal dependendo do nível de consciência e colaboração do paciente. Dependendo das propriedades e do mecanismo pulmonar, a pressão pleural pode ultrapassar a atmosférica durante a inspiração, a força de contração do pulmão e da parede torácica armazenada como energia potencial na inspiração com pressão positiva causando uma expiração passiva, A execução da técnica pode durar de 15 a 20 minutos, dependendo da sua indicação, sendo aplicada pelos aparelhos como Bird, Mark 7, o Bennet AP-5B e o reanimador de Muller, entre outros acoplados a mascaras. O fisioterapeuta vai ajustar a pressão inspiração máxima de acordo com cada paciente que deve permitir o início da inspiração com esforço mínimo e o fluxo baixo a moderado para permitir inspiratório mais longo possível (Musseti, et al, 2006).

\subsection{Espirometrias de incentivo}

Na espirometria de incentivo (EI) são utilizados aparelhos que por meio de estímulos visuais/ e ou auditivos incentiva o esforço inspiratório. O seu principal efeito e o aumento da expansão pulmonar por meio de uma pressão negativa, consequentemente elevando a pressão transpulmonar e promovendo melhor fluxo de gás entre a via aérea e os alvéolos. É fundamental a participação do paciente, que deve realizar uma inspiração lenta e profunda, desde a capacidade residual final até capacidade pulmonar total sustentando de dois a três segundos. A quantidade exata de inspiração máxima sustentada não é conhecida e vai variar de acordo com o paciente e sua condição clínica.

É importante que durante a EI enfatize a expansão do tórax inferior para evitar o uso da musculatura acessória. Essa é uma recomendação importante principalmente para os pacientes de PO de cirurgia cardíaca tem a tendência a realizar a respiração 
apical, e para prevenir uma hiperventilação e consequentemente causar uma hipercapnia, sendo assim deve ser realizado um repouso de 30 segundos a 1 minutos entre cada inspiração. É recomendado que o tempo inspiratório seja longo e volume elevado.

Os aparelhos podem ser orientados pelos fluxos ou pelo volume. Os de fluxo são possíveis visualizar e calcular o fluxo inspirado. Os de volume apresenta um dispositivo que possibilita administrar o fluxo, assim possibilitando a monitorização e otimização dos dois avaliando a eficácia da terapia (O volume alto e o fluxo baixo), (Vera Lúcia. et al., 2006).

\subsection{Pressões positiva continua nas vias aéreas (CPAP)}

É definida como uma pressão positiva continua durante todo o ciclo respiratório, pode ser fornecido por meio não invasivo, com auxílio da máscara nasal, bucal, facial ou total. Começou a ser utilizada em no final de 1970 a início de 1980, por Poulton e Oxon que fazia uso do CPAP para reexpanção pulmonar em pacientes com edema agudo de pulmão. Com o CPAP e possível diminuir a frequência respiratório, aperfeiçoar a capacidade vital, melhora da ventilação-minuto e aumentar a capacidade residual final. A utilização do CPAP vem sendo muito efetivo nos PO de cirurgia cardíaca (tratando as insuficiências respiratórias agudas e prevenindo e tratando complicações respiratórias).

A funcionalidade dessa técnica consiste no fornecimento do gás inspirado por meio de uma única pressão de fluxo aéreo interrupto. Durante a inspiração espontânea é fornecido um fluxo inspiratório continuo, assim facilita a inspiração e consequentemente reduz o trabalho respiratório. E no decorre da expiração é mantida uma PEEP de 5 a 20 CMH20, com intuído de facilitar o recrutamento dos alvéolos colapsados e do aumento da capacidade da capacidade residual funcional. A regulagem e o tempo de aplicação vão depender do estado clinico de cada paciente, em paciente em PO vai ter uma diferença em relação ao objetivo da terapia seja ela curativa (tratar insuficiência aguda) ou profilática (prevenir distúrbios respiratórios), sendo assim sendo de suma importância realizar uma avaliação minuciosa com o paciente, para definir melhores parâmetros necessários no tratamento (Guizilini, et al., 2006).

\subsection{Pressões positiva expiratória nas vias aéreas (EPAP)}

É considerada a técnica que melhor apresenta custo benefício, pois é de fácil manuseio e de baixo custo de fornecer PEEP (Pressão positiva no final da expiração) em respiração espontânea.

A execução da EPAP se dá por uma inspiração acompanhada de uma expiração resistida e lenta, ocasionando assim, uma pressão positiva nos alvéolos, e fazendo com que o volume do alvéolo se mantenha por mais tempo, evitando o colabamento. Para melhor eficácia do equipamento é importante saber escolher a máscara adequada para os pacientes, ela deve ser confortável e adequada ao formato do rosto, e a máscara pode ser presa por uma presilha ou pode ser posicionada manualmente pelo terapeuta. A quantidade de aplicação vai depender do paciente e sua capacidade de prosseguir com o método, mais geralmente é realizada de duas a quatro vezes ao dia.

O uso do PEEP associado a exercício de respiração profunda traz muito benefício entre eles está o aumento da demanda respiratória, a melhora da recuperação da função pulmonar, foi bastante efetiva na recuperação de força muscular respiratória e a diminuição de áreas com atelectasia (Musetti, et al., 2006).

\subsection{Pressão positiva em dois níveis (BiPAP/ BELIVEL)}

É um método espontâneo onde apresenta dois níveis de pressão, o IPAP (Pressão Inspiratória Positiva nas Vias Aéreas) e o EPAP (Pressão expiratória positiva na Vias Aéreas), e proporciona ao paciente o suporte nos dois ciclos respiratórios. Fazendo com que tenha uma relação entre as fases semelhante à relação Inspiração X Expiração. A utilização do BiPAP vem se mostrando ter mais benefícios que o CPAP, por diminuir as áreas com atelectasia e reduzir o shunt (sangue que entra no leito arterial sistêmico sem passar pelas áreas ventiladas do pulmão). A forma de ajuste, duração e o intervalo de utilização desse equipamento 
podem variar de acordo com as condições clinicas em que o paciente se encontra. Nos pacientes pós-operatório o objetivo de aplicação (curativo ou profilático) pode estar diretamente relacionando aos parâmetros que devem ser aplicados (Musetti, et al., 2006).

\section{Conclusão}

O presente estudo demonstra que a cirurgia cardíaca provoca várias alterações pulmonares, renais, neurológicas, sendo comum ocorrer cerca de 70\% dos casos. De acordo com os estudos analisados, a aplicação das técnicas de reexpansão pulmonar no pós operatório de cirurgia cardíaca se mostrou relevante na melhora do quadro funcional desses pacientes, promovendo uma melhor ventilação pulmonar expandindo os tecidos pulmonares colapsados, promovendo uma ventilação perfusão adequada os tecidos e melhorando a função cardiovascular sendo assim reduzindo a internação, amenizando e/ou prevenindo as complicações pós operatórias. A fisioterapia respiratória é fundamental no controle dos cuidados do paciente no pré e no pós-operatório pois colabora consideravelmente para um melhor prognostico. Considera-se de suma importância a atuação da fisioterapia respiratória e a execução das técnicas de reexpansão pulmonar no pré e pós-operatório de cirurgia cardíaca, porem verifica-se a necessidade de novos estudos sobre a aplicação das técnicas de reexpansão pulmonar que mais beneficiam esses pacientes no meio hospitalar, na tentativa de padronizar esses procedimentos.

\section{Referências}

Arcênio, L, Souza, M. D, Bortolin, B. S, Fernandes, A. C. M, Rodrigues, A. J., \& Evora, P. R. B. (2008). Cuidados pré e pós-operatórios em cirurgia cardiotorácica: uma abordagem fisioterapêutica. Rev. Bras. Cir. Cardiovasc. 23(3), 400-410.

Azeredo, C. A. C (2000). Fisioterapia respiratória moderna. (2a ed.), Manole.

Azeredo, C. A. C. (2002). Fisioterapia respiratória no hospital geral. Manole.

Barros, G. F., Santos, C. S., Gramado, F. B., \& Costa, P. P. (2010). Treinamento muscular respiratório na revascularização do miocárdio. Rev. Bras. Cir. Cardiovasc.; 25(4), 483-490.

Cavenaghi, S, Ferreira, L. L, Marino, L. H. C, \& Lamari, N. M (2011). Fisioterapia Respiratória no pré e pós-operatório de cirurgia de revascularização do miocárdio. Rev. Bras. Cir. Cardiovasc.; 26(3), 455-61.

Corrêa, P. R., Catai, A. M., Takakura, I. T., Machado, M. N., \& Godoy, M. F. (2010). Variabilidade da frequência cardíaca e infecções pulmonares pósrevascularização miocárdica. Arq Bras Cardiol.

Dias, C. M.; Vieira, R. O.; Oliveira, J. F.; Lopes, A. J. et al. (2011). Três protocolos fisioterapêuticos: Efeitos sobre os volumes pulmonares após cirurgia cardíaca.J. Bras. Pneumol; 37(1), 54-60.

Feltrim M. I. Z., \& Parreira V. F. 1994 á 2000, (2001). Fisioterapia Respiratória: Consenso de Lyon.

GIL, A. C. (2002). Como elaborar projetos de pesquisa. Atlas.

Leguisamo C. P, Kalil, R. A. K., \& Furlani, A. P. (2005). A efetividade de uma proposta fisioterapêutica pré-operatória para cirurgia de revascularização do miocárdio. Rev Bras Cir Cardiovasc.

Lima, P. M. B, Cavalcanti, H. E. E, Rocha, A. R. M, \& Brito, R. T. F. (2011). Fisioterapia no pós-operatório de Cirurgia cardíaca: a percepção do paciente. Rev. Bras. Cir. Cardiovasc. 26(2), 244-9.

Nicolau J. C. (2010). Condutas práticas em cardiologia. p. 914 Manole.

Overend, T. J., Anderson, C. M., Lucy, S. D., Bhatia, C., \& Jonsson, B. I. C. (2001). TimmermansC. The effect of incentive spirometry on postoperative pulmonar complications: a systemactic review.

Peixoto, R. S., Pires H. R, Rosa Netto, M. V., Pena, F. M, Areas, G. S, Moraes, F. V. D., et al. (2009) Revascularização Miocárdica no Idoso. Rev SOCERJ.

Pryor J. P., \& Webber, B. A. (2002) Fisioterapia para problemas respiratórios e cardíacos. (2a ed.), Guanabara/ Koogan.

Renault, J. A., Costa-Val, R., \& Rossetti, M. B. (2008). Fisioterapia respiratória na disfunção pulmonar pós-cirurgia cardíaca. Rev. Bras. Cir. Cardiovasc.; v.23 n.4 p: 562-569, Belo Horizonte.

Machado, M. da G. R. (2021). Bases da fisioterapia respiratória: terapia intensiva e reabilitação. Guanabara Koogan.

Scarlan C. L, Wilkins R., \& Stoller J. K. (2011). Fundamentos da Terapia Respiratoria de Egan. (7a ed.), Editora Manole Ltda. 
Research, Society and Development, v. 10, n. 12, e73101220143, 2021

(CC BY 4.0) | ISSN 2525-3409 | DOI: http://dx.doi.org/10.33448/rsd-v10i12.20143

Tarantino, A. B. et al. (2002). Doenças pulmonares. Guanabara Koogan.

Titoto L., Sansão M. S, Marino L. H. C, et al. (2005). Reabilitação de pacientes submetidos à cirurgia de revascularização do miocárdio: atualização da literatura nacional. 12(4), Dez Arquivo Ciência Saúde.

West J. B. (1990). Fisiologia Respiratoria Moderna. (3a ed.), Editora Manole Ltda. 